Editorial

\title{
An HIV view of the human condition
}

Although there have been some significant setbacks, our achievements have been impressive and we are guardedly optimistic for the future (we refer to ourselves as 'we' because there are several strains of HIV viruses). Since humanity first became aware of our existence in 1981, we have made major inroads, with about 19 million infected humans most, if not all, of whom will eventually develop significant illnesses and AIDS, which is a uniformly fatal syndrome. We are thus the most successful member of the retrovirus club, the elite family of viruses which defied previous human dogma in that our sequences can be changed from RNA to DNA. We are also able to integrate with our host's genetic information.

Our origin remains obscure. We were almost certainly an asymptomatic infection of primates which entered the human population in Africa and thereafter spread unrecognised in the indigenous human population. Infected individuals remained well but infectious and from then on we spread unsuspected around the world. Initially we were perceived as an American disease which affected a minority group (male homosexuals). It was only later that these individuals became symptomatic and revealed our activities and later still that our wider potential was appreciated.

We particularly attack the human immune system, especially T-cell-mediated immunity, which makes humans vulnerable to attack by intracellular pathogens. We attack the humoral immune system, creating chaos among B lymphocytes, which produce large amounts of useless antibodies, making infected individuals vulnerable to attack by extracellular pathogens. We also attack the central nervous system, so that, even if immune system damage were minimised, we could cause major neurological illness, notably dementia. Reduction of viral load elsewhere in the body may reduce the chances of this, but poor penetration of anti-HIV drugs into the brain might possibly allow virus based in the central nervous system to become resistant. Time will tell.

We are a primarily a Sexual Transmitted Disease (STD) which has given us three main advantages. Firstly, as delicate organisms, we have avoided much wastage by discovering a route of transmission that avoids harsh external environments. Secondly, there is a lot of human sexual activity going on. There is one new attendance at STD clinics for every 56 members of the UK population above the age of 15 and below retirement age each year. Of course, not all STD clinic attenders have an STD, but still that is an awful lot of humans who think they might have been at risk of STDs. Thirdly, human semen is immunosuppressive (presumably to protect the foreign protein spermatozoa from being killed by female immune reactions), presenting a highly satisfactory vehicle in which to transport an infection. No doubt our infecting dose is greatly reduced by presentation in semen, but humans have not done the relevant studies - lack of volunteers presumably.

Humanity has even ignored warnings! A British author, HG Wells, wrote a 'history' of future developments The shape of things to come in 1933. In this he detailed the maculated fever which was 'hitherto known only as a disease of captive baboons' and 'all who took it died' (the macule anticipating Kaposi's sarcoma). He brilliantly anticipated the spread of asymptomatic HIV infections which would later develop into AIDS itself. 'The real disease... may not have been the maculated fever at all, but the state of vulnerability that had spread unsuspected throughout the world'.

\section{Strengths}

Our long incubation period (on average in the untreated, it is about 10 years before HIV leads to AIDS) combined with our (admittedly variable) infectivity, ensures that the average HIV-infected person transmits infection to more than one other person, thus ensuring perpetuation of the world HIV epidemic.

We have allowed numerous other human pathogens to flourish. The damage we inflict on cell and humoral human immune systems offers further advantage to our non-HIV microbial colleagues by masking the presence of other diseases by disabling those normal human immune responses to infection which are necessary to produce classical symptoms and signs by which humans traditionally make diagnoses. The damage to the human immune system also ensures that many diagnostic tests which depend upon an immune system response are invalidated. Notable amongst the colleagues we have helped are Mycobacterium tuberculosis (a bacteria) and cytomegalovirus (a virus). We can also induce tumours such as lymphoma and Kaposi's sarcoma, the latter being associated with infection with a herpes virus.

We have displayed an awesome ability to incur costs for human society. Aside from the deaths, the financial burdens related to healthcare are prodigious. Investigations and treatments are nearly all recently developed and expensive. Currently, humans disagree as to when anti-HIV treatment should be commenced but there is consensus that at least two (expensive) drugs should be used in combination in the long term. A severe financial burden for any healthcare system! And effective control measures to combat HIV infection by containment cannot be introduced in a free society (assuming there were any which worked).

There is no chance that Darwinian evolution will lead to our downfall. Humans will not breed resistant individuals. Darwinian natural selection depends on premature death before reproduction but many HIV-infected humans will have reproduced before they die. We have even been able to elicit the help of some human allies, who still dispute that HIV causes AIDS. We find this strange but encouraging. Some of these allies point out, absolutely correctly, that a few AIDS patients have no evidence of HIV infection. This is a statement of the obvious. AIDS is a syndrome and as such is a collection of symptoms and signs which does not define a single aetiology. It would have been better for humans if AIDS had been called HIV-associated immunodeficiency. Although prior to this statement we viruses have neither spoken nor written, there is no doubt that arguments about words have delayed progress countering our achievements!

We have challenged fundamental aspects of human society. In developed countries, injection drug users have held society to ransom by insisting upon their right to inject drugs. Because society has deemed such activity criminal, these people have been imprisoned in circumstances where easily concealed drugs can be smuggled in. As needles and syringes are more difficult to smuggle, this situation is perfect for needle sharing, which greatly aids the spread of HIV (and incidentally that of hepatitis B and C). Each 
human being with severe HIV costs society about $£ 16000$ a year, one with AIDS about $£ 31000$, and treatment of the asymptomatically infected looks set to cost about $£ 10000$. Which human societies can cope with this, when long-term survival becomes a possibility? Can the world afford a bill of, say, $£ 10000$ times 19 million each year?

Humans have attempted to mount health education programmes which have been variably effective. Warnings about AIDS must be given to children before they are likely to indulge in at-risk activities and this means telling them about activities about which some of them would hithertofore have been ignorant. Describing AIDS as an anxietyprovoking deterrent against at-risk activities acts only in the short-term, however, and the information given may encourage children to think that at-risk activities must be interesting (as some undoubtedly are).

\section{Threats}

\section{ANTI-HIV DRUGS}

Trials of drugs targeted at us are fraught with problems for humans. Evaluation of any anti-HIV drug should involve long-term trials. Currently, many drugs are being introduced after short-term trials have shown benefit on surrogate markers of clinical improvement (CD4 counts, viral load, etc) or actual clinical improvement, but the long-term sideeffects of such treatments are not known. The imminent ultra-long-term HIV suppressive treatments will reveal unexpected side-effects because nearly all such drugs affect human genetic material (protease inhibitors are one exception). Additionally, all anti-HIV drugs are essentially viristatic and not viricidal and we will have time to develop resistance. Admittedly development of resistance to triple therapy will be difficult, but the achievements of our mycobacterial cousins in developing resistance to drugs gives rise to optimism.

It will be decades before humans develop drugs that can penetrate every HIV-infected cell in the human body to selectively remove HIV-directed nucleic acid sequences from the host cell genome. Such a drug would need to be a highly specific, targeted, guided missile, far superior to Ehrlich's magic bullets, which rather crudely damage our much larger cousins, the bacteria. Our ability to mutate will make it difficult to develop uniformly effective broadspectrum anti-HIV drugs (and even more difficult to develop an effective vaccine).

Nevertheless, humanity is fighting back. Numerous drugs are being developed, trials are being performed of drugs, and combinations of drugs, in different genetic groups, in different geographic areas, and with different strains of HIV. After all the data are analysed, using sophisticated euphonious statistical methods, humans make the assumption that one set of statistically significant results in one set of patients from one area necessarily applies elsewhere. We wonder how correct this assumption is.

\section{ANTI-HIV VACCINES}

Humans have a naive belief that a vaccine will be the answer for every infection, but HIV possesses attributes which will minimise this threat. There are six reasons for optimism: firstly, the human response to natural HIV infection is only partially effective (and probably operates to our benefit by prolonging our incubation period so that there is a longer time during which transmission might occur). Any antiHIV vaccine will have to evoke host responses many many times more effective than the usual host response. Secondly, no vaccine is perfect. Vaccination before infection might not be $100 \%$ preventative but only prolong our incubation period. Vaccination after infection, aimed at boosting host immunity to suppress us further, will certainly prolong it. Thirdly, killed vaccines are in general less effective than live vaccines. Because of our propensity to develop mutations, no one would dare give a live attenuated HIV-derived vaccine. Fourthly, if HIV were common in a particular population then all members of the human population would have to be vaccinated before they entered into at-risk activities: in other words, a routine vaccination in childhood. No vaccine is free of side-effects, however, and any routine vaccination of childhood will have to have negligible side-effects if uptake is to be high. Fifthly, an infallible test to differentiate vaccine-induced immune responses from natural-infection-induced immune responses would have to be developed (and if HIV vaccination were a routine childhood vaccination then all bloodderived products would have to be thus screened). And finally, immunity conferred by most vaccines acts best against pathogens that spread by means of body fluids. However, we can spread directly from cell to cell without being exposed to potentially harmful antibodies (or drugs) in body fluids.

Humans will also have to answer five questions on HIV vaccination: Could one vaccinate the whole of Africa, the major reservoir of HIV infection? How long would vaccine immunity last? If not of long duration, how often would vaccination have to be repeated? Would the vaccine protect against all HIV strains arriving by any of the possible routes of infection? And last, does the vaccine cut down infectivity for those who are vaccinated as well as for those who are infected?

Finally, the Public Health Paradox. Some people who are vaccinated against HIV may think themselves immune and indulge in more at-risk activity. As no vaccine (except perhaps for Yellow fever) offers complete immunity, given a high enough infecting dose, any infection (including HIV) could overcome vaccine-induced responses.

Thus, we conclude that vaccination might even increase spread of HIV infection. In any case, we have many years before a vaccine will be a significant challenge to us. Let us be realistic. Even if humans managed to develop a vaccine that offered $100 \%$ protection, was cheap, conferred longlasting immunity, and had no side-effects, how long would it take to evaluate and vaccinate the world? Several years at least, during which we can progress and evolve. And humanity will still have the cost of looking after those already infected.

\section{Weaknesses}

Modesty demands that we confess that our only serious failing is that we cannot be spread by the aerosol route.

\section{DISCLAIMER}

Although we are seemingly perfectly designed to attack human populations, we deplore allegations that we were designed ('genetically engineered') in, and escaped from, biological laboratories: the technology to create us was not available when we first entered the human population (probably sometime before 1959).

\section{Conclusions}

When a future HG Wells is writing the history of the 20th Century, HIV will be deemed to be more significant than many wars.

Text of a report leaked to the Postgraduate Medical fournal by Philip D Welsby, Department of Infectious Diseases, The City Hospital, Edinburgh EH10 5SB, UK 